\title{
Lösungsmöglichkeiten nach fehlgeschlagenen Osteosynthesen am distalen Radius
}

\author{
Christian Voigt, Norbert Gärtig
}

\section{Zusammenfassung}

Sekundäre Dislokationen nach distalen Radiusfrakturen entstehen nach konservativer Therapie mit unzureichender Retention des Repositionsergebnisses und nach Versagen von (häufig falsch angewandten) Osteosynthesen. Hier ist besonders die Bohrdrahtosteosynthese zu nennen. Bei der palmaren Dislokation („Smith fracture") ist die Anwendung von Bohrdrähten ein Fehler, die palmare
Platte ist seit Jahren in diesen Fällen indiziert. Jede größere Fehlstellung muss korrigiert werden, auch beim älteren Patienten - unweigerlich folgen sonst Schmerzen und Funktionsverlust des Handgelenks oder gar der Hand. Als Verfahren der Wahl sollte eine Plattenosteosynthese nach offener Reposition durchgeführt werden, bei Defekten ist die Indikation zur Auffüllung mit autologer Spongiosa oder Knochenersatzmaterial gegeben.

\section{Einleitung}

Die distale Radiusfraktur beim Erwachsenen ist eine häufige Verletzung. Die Wahl des Therapieverfahrens (konservativ versus operativ) unter Berücksichtigung der Frakturform ist entscheidend für den Heilungsverlauf und damit für die Funktion des Handgelenkes nach knöcherner Konsolidierung der Fraktur. Trotz der Häufigkeit der distalen Radiusfraktur gibt es keine verbindlichen Therapieempfehlungen, da signifikante Studien fehlen. Mit adäquater Therapie heilt ein großer Teil der Radiusfrakturen zufrieden stellend aus. Pseudarthrosen entwickeln sich im Bereich des distalen Radius nur sehr selten. Probleme ergeben sich in der Behandlung von sekundär dislozierten Radiusfrakturen sowohl nach konservativer aber auch nach operativer Versorgung. Wir möchten in diesem Artikel einige Fälle von sekundär dislozierten Radiusfrakturen bzw. Fälle nach fehlgeschlagenen Osteosynthesen und deren Therapie darstellen.

OP-JOURNAL 2004; 20: 112-114

(c) Georg Thieme Verlag KG Stuttgart · New York

\section{Therapieversager nach primär konservativem Vorgehen}

Metaphysäre nicht dislozierte distale Radiusfrakturen ohne Gelenkbeteiligung können primär konservativ behandelt werden. Dislokationen im Sinne der typischen Extensionsfraktur neigen aufgrund einer dorsalen Trümmerzone nach Reposition häufig zum „Nachsintern“, insbesondere dann, wenn eine kortikale Abstützung fehlt. Die Sinterung (Längenverlust des Radius und Abflachung des Radiusbasiswinkels sowie der palmaren Neigung der Gelenkfläche in der Seitansicht) führen bei Ausheilung in dieser Position zu erheblichen Problemen im Handgelenk in Form von Bewegungseinschränkung, Schmerzen und sichtbarer Fehlstellung. Außerdem führt die Störung des distalen Radioulnargelenks zu Problemen in der Umwendbewegung des Unterarms, der relative Ellenvorschub auch zum Ulna Impaction Syndrom. Dieser Repositionsverlust kann sowohl während der Ruhigstellung im Gipsverband als auch noch nach Abnahme des Gipsverbandes eintreten.

Therapeutisch sollte in einem solchen Fall die offene Nachreposition und eventuell auch die Defektauffüllung mittels autologer Spongiosa oder Knochenersatzstoffen und die Stabilisierung durch eine Plattenosteosynthese erfolgen.

Als Beispiel für einen solchen Fall sind in Abb.1a und b die Röntgenbilder einer 6 Wochen alten, in Fehlstellung konsolidierten distalen Radiusfraktur dargestellt. Operativ führten wir über einen dorsalen Zugang die offene Reposition und Defektauffüllung mittels kortikospongiösem Span aus dem Beckenkamm sowie die Stabilisierung mit einer winkelstabilen dorsoulnaren Platte $2,4 \mathrm{~mm}$ und einem Kirschner-Draht durch. Die postoperativen Bilder sind in den Abbildungen 1c und d dargestellt.

Die Abb. 2a bis d zeigen die Rettung der Situation 3 Wochen nach unverständlicherweise begonnener konservativer Therapie mit Osteosynthese von palmar mittels $2,4 \mathrm{~mm}$ winkelstabilem Implantat und zusätzlichem Bohrdraht von ulnar zur Erhöhung der primären Stabilität.

Insgesamt sind Korrektureingriffe bei in Fehlstellung verheilten distalen Radiusfrakturen nach konservativer Therapie selten, da ein Großteil der Patienten trotz radiologisch nachgewiesener Fehlstellung zunächst kaum Beschwerden hat und die Beweglichkeit im Handgelenk bei diesen Patienten meist nur endgradig eingeschränkt ist. Nicht selten wird auch von weiterbehandelnden Ärzten auf das üblicherweise fortgeschrittene Lebensalter der Patienten verwiesen und unterstellt, dass die erzielte Stellung und Funktion ausreiche. Diese Situation kann natürlich nicht befriedigen, die möglichst exakte Wiederherstellung nach Fehlheilung sollte auch beim Älteren angestrebt werden.

\section{Therapieversager nach primär operativem Vorgehen}

Ein weiterer Grund für Korrekturoperationen kann bei Repositionsverlust nach primärer Kirschner-Drahtosteosynthese bestehen. Retrospektiv ist als Ursache 


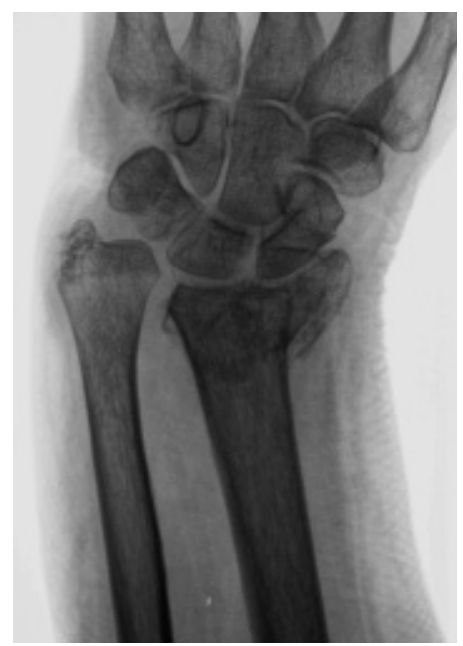

Abb.1a,b 6 Wochen nach Einleitung einer konservativen Therapie mit diesem Unfallbild Zuweisung des Patienten.
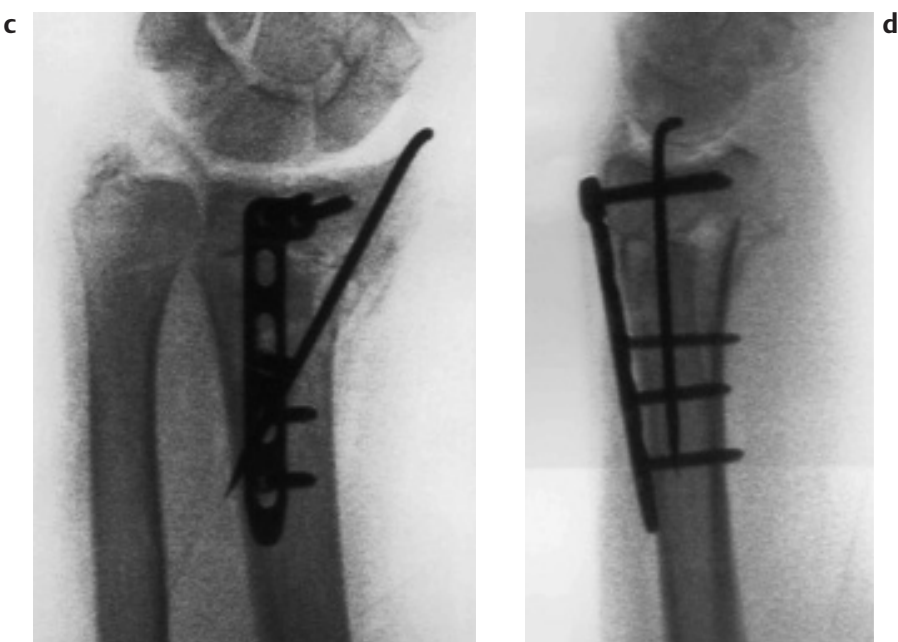

Abb.1c, d Korrektur mit autologem Spongiosablock und Platte $2,4 \mathrm{~mm}$ von dorsal.

a

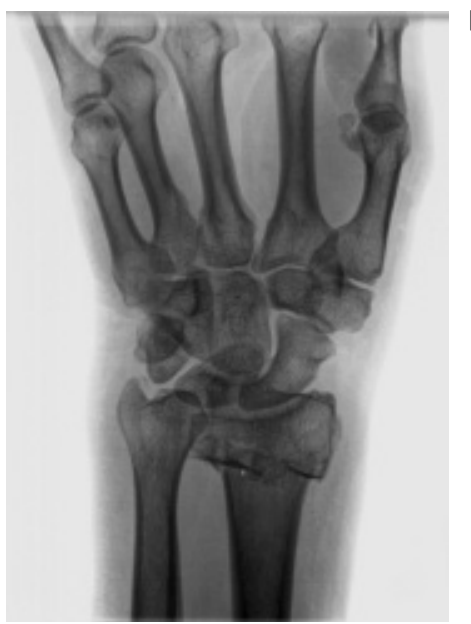

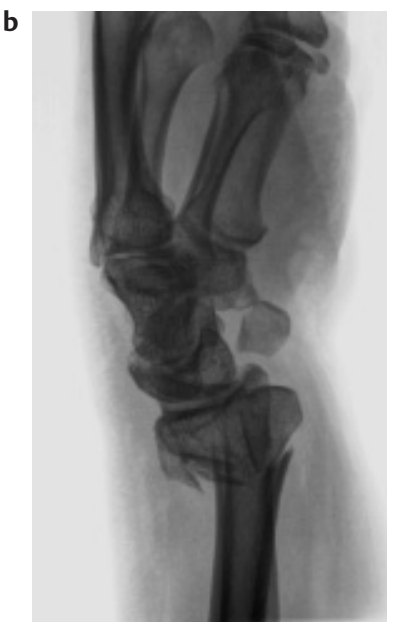

Abb. 2a, b Primäre konservative Therapie dieser A2-Fraktur. c
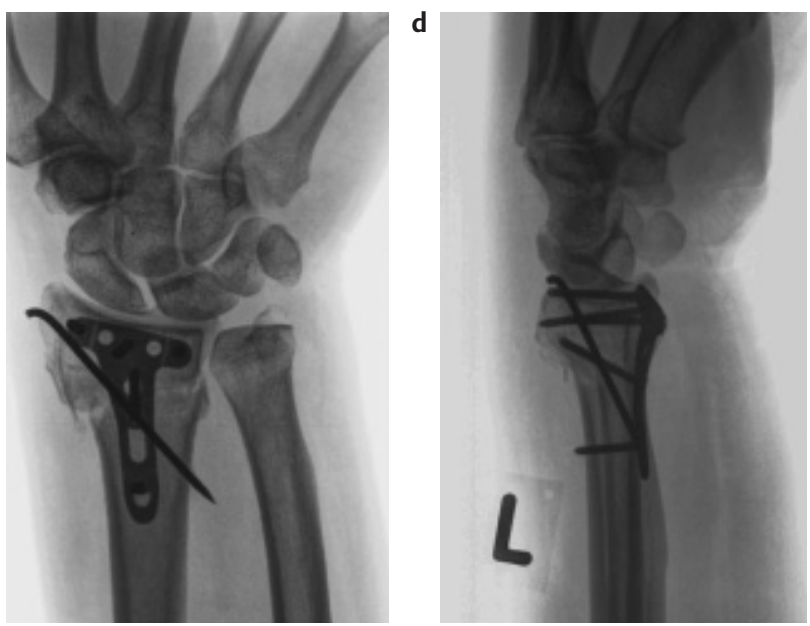

Abb.2c, d Korrekte Osteosynthese mit 2,4 mm Implantat und zusätzlich stabilisierendem Kirschner-Draht. dafür meist ein primär falsches Einschätzen der Frakturmorphologie beim Entschluss zur primären Kirschner-Drahtosteosynthese zu sehen. Die KirschnerDrahtosteosynthese erzielt in solchen Fällen keine ausreichende Stabilität bzw. einzelne Fragmente wurden oder konnten aufgrund ihrer Lokalisation nicht erfasst werden. Wenn diese Frakturen auch noch mit einer Gelenkbeteiligung einhergehen, führen sie bei Ausheilung in einer solchen Fehlstellung $\mathrm{zu}$ einer erheblichen Bewegungseinschränkung im Handgelenk und entsprechenden Beschwerden. Die Dislokation lässt sich in der Regel bereits in der 1. Woche nach Kirschner-Drahtosteosynthese radiologisch nachweisen. Die Therapie der Wahl in einem solchen Fall ist in unseren Augen die offene Reposition - je nach Frakturmorphologie über einen dorsalen oder palmaren Zugang - und Abstützung der Fraktur mittels $2,4 \mathrm{~mm}$ winkelstabilen Platten ggf. in Kombination mit Kirschner-Drähten. Bei ausgedehnter Trümmerzone erfolgt auch hier eine Spongiosaplastik oder Einbringen von Knochenersatzmaterialien zur Defektauffüllung. Häufig lässt sich in solchen Fällen eine relativ stabile Situation erzielen, so dass die postoperative Gipsbehandlung dadurch erheblich verkürzt werden kann. Die Abb. 3a bis f zeigen die unkorrekte Osteosynthese einer Flexionsfraktur mit typischer palmarer Dislokation und die Korrektur des Dilemmas mit 2,4 mm winkelstabiler Platte von palmar. In $\mathbf{A b b}$. $\mathbf{4 a}$ bis $\mathbf{c}$ wird die Problemlösung bei gleichem Fehler mit konventioneller 3,5 mm T-Platte dargestellt. 

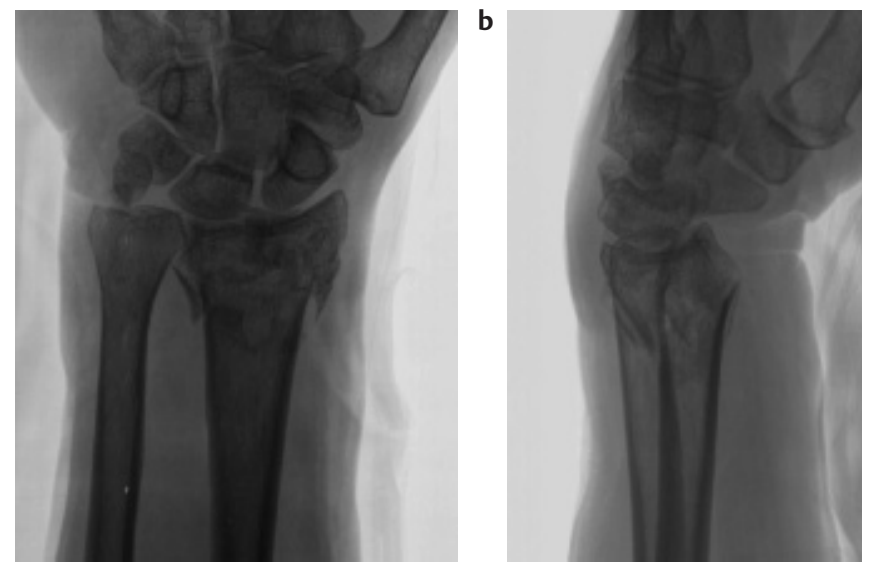

Abb.3a,b Typische palmare Dislokation einer „Smith-fracture.“
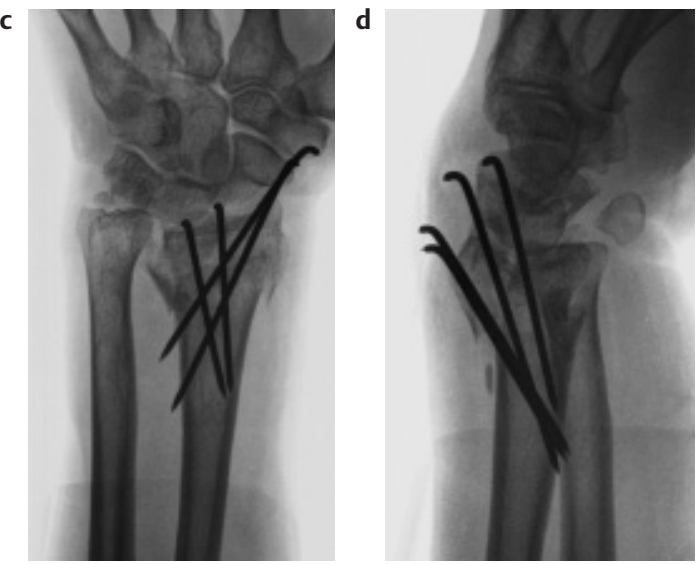

Abb. 3c, d Fehlerhafte Osteosynthese mit Bohrdrähten und erwartungsgemäßer Redislokation nach palmar.
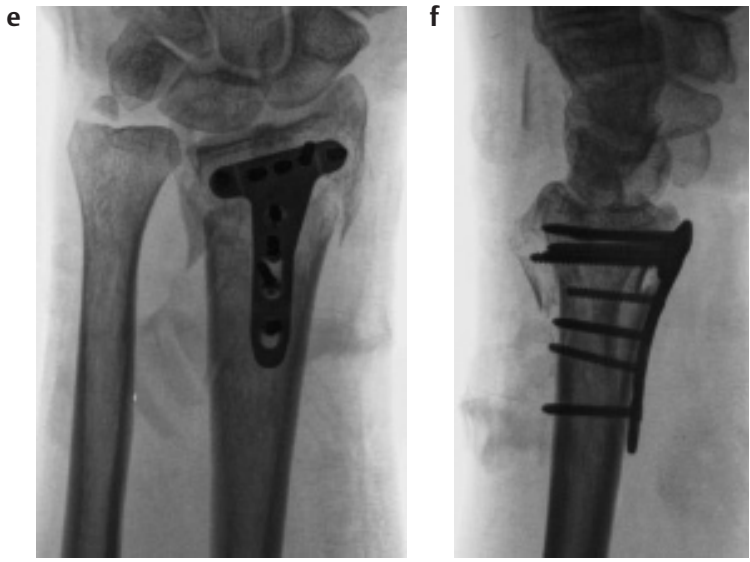

Abb.3e,f Regelrechte Versorgung mit Plattenosteosynthese von palmar (2,4 mm Platte winkelstabil).
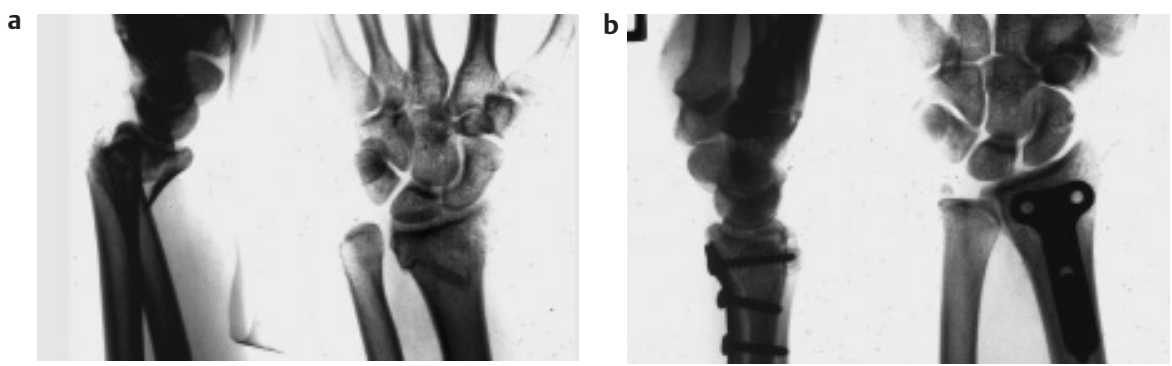

Abb. 4a, b Gleiche Situation wie in Abb. 3, Problemlösung mit konventioneller 3,5 mm Platte.

Implantatversagen bei distalen Radiusfrakturen in Form von Plattenbrüchen oder Plattenausbrüchen haben wir in unserem eigenen Krankengut in den letzten Jahren nicht gesehen. Gelegentlich kommt es nach Kirschner-Drahtosteosynthese zu einem Wandern der Drähte.
Die Ursache hierfür war in diesen Fällen immer das fehlende Umbiegen der Drähte an ihrem Ende. Die Drahtwanderung trat meist erst bei bereits fast vollständig konsolidierter Fraktur auf, so dass sich daraus bei uns keine sekundäre Frakturdislokation ergab.

\section{Schlussfolgerung}

Die in Fehlstellung verheilte bzw. sekundär dislozierte Radiusfraktur muss therapiert werden, wenn eine deutliche Achsfehlstellung, eine deutliche Radiusverkürzung sowie eine Gelenkstufenbildung vorliegt. Je jünger der Patient ist, desto konsequenter muss vorgegangen werden. Der alte Patient enthebt uns aber nicht von der Pflicht zum Handeln. Therapeutisch wird dann in der Regel eine offene Reposition und Plattenosteosynthese ggf. mit Spongiosaplastik bzw. Knochenersatzmaterialien zur Defektauffüllung durchgeführt. Mit diesen Verfahren lassen sich in der Regel ein gutes funktionelles Ergebnis sowie eine problemlose Konsolidierung der Fraktur erzielen.

\section{Literatur}

1 Tscherne Unfallchirurgie: Ellbogen, Unterarm, Hand Band 1 Ellbogen, Unterarm: K.P. Schmit-Neuerburg, H. Towfigh, R. Letsch (Herausgeb.) Springer Berlin Heidelberg New York 2001

Prof. Dr. med. Christian Voigt Chefarzt

\section{Dr. med. Norbert Gärtig}

Oberarzt

Klinik für Unfall-, Hand- und Wiederherstellungschirurgie Städtisches Klinikum Solingen Gotenstr. 1

D-42653 Solingen 